\title{
Structure and Outcomes of Interdisciplinary Rounds in Hospitalized Medicine Patients: A Systematic Review and Suggested Taxonomy
}

\author{
V. Surekha Bhamidipati, MD ${ }^{1,2 *}$, Daniel J. Elliott, MD ${ }^{1,2}$, Ellen M. Justice, MLIS, AHIP ${ }^{3}$, Ene Belleh, MLS, AHIP, \\ Seema S. Sonnad, $\mathrm{PhD}^{2}$, Edmondo J. Robinson, MD, MBA',2
}

${ }^{1}$ Department of Medicine, Christiana Care Health System, Wilmington, Delaware; '2Value Institute, Christiana Care Health System, Wilmington, Delaware; ${ }^{3}$ Medical Libraries, Christiana Care Health System, Wilmington, Delaware.

BACKGROUND: Interdisciplinary rounds (IDR) have been described to improve outcomes. However, there is limited understanding of optimal IDR design.

PURPOSE: To systematically review published reports of IDR to catalog types of IDR and outcomes, and assess the influence of IDR design on outcomes.

DATA SOURCES: Ovid MEDLINE, Ovid MEDLINE InProcess \& Other Non-Indexed Citations, Journals Ovid, Cumulative Index to Nursing and Allied Health Literature (EBSCOhost), and PubMed from 1990 through December 2014, and hand searching of article bibliographies.

STUDY SELECTION: Experimental, quasiexperimental, and observation studies in English-language literature where physicians rounded with another healthcare professional in inpatient medicine units.

DATA EXTRACTION: Studies were abstracted for study setting and characteristics, and design and outcomes of IDR.
DATA SYNTHESIS: Twenty-two studies were included in the qualitative analysis. Many were of low to medium quality with few high-quality studies. There is no clear definition of IDR in the literature. There was wide variation in IDR design and team composition across studies. We found three different models of IDR: pharmacist focused, bedside rounding, and interdisciplinary team rounding. There are reasonable data to support an association with length of stay and staff satisfaction but little data on patient safety or satisfaction. Positive outcomes may be related to particular components of IDR design, but the relationship between design and outcomes remains unclear.

CONCLUSIONS: Future studies should be more deliberately designed and fully reported with careful attention to team composition and features of IDR and their impact on selected outcomes. We present a proposed IDR definition and taxonomy for future studies. Journal of Hospital Medicine 2016;11:513-523. (C) 2016 Society of Hospital Medicine
Interdisciplinary rounds (IDR) constitute a model of care where healthcare team members representing multiple disciplines meet to develop patient care plans. IDR allow input from a range of professionals without communication lag, thereby improving communication while incorporating diverse sets of information. IDR appear to improve collaboration among physicians and nurses, ${ }^{1}$ increase compliance with guidelines, ${ }^{2}$ improve safety and quality, ${ }^{3}$ reduce adverse drug events, ${ }^{4}$ and possibly lower mortality. ${ }^{5}$ Recommendations have been published regarding implementation of IDR. ${ }^{6}$ The Institute for Healthcare Improvement (IHI) supports IDR as a formal daily mechanism for identifying patient safety risks and determining daily goals. ${ }^{7}$ IHI recommendations include guidance on team membership, patient and family participation, using a daily goals sheet, and

*Address for correspondence and reprint requests: Surekha V. Bhamidipati, MD, 200 Hygeia Dr., Newark, DE 19713; Telephone: 302-733-

5613; Fax: 302-623-0554; E-mail: sbhamidipati@christianacare.org

Additional Supporting Information may be found in the online version of this article.

Received: September 24, 2015; Revised: December 14, 2015; Accepted: December 22, 2015

2016 Society of Hospital Medicine DOI 10.1002/jhm.2575

Published online in Wiley Online Library (Wileyonlinelibrary.com). addressing safety concerns. However, there is no standard definition of IDR. Consequently, there is variation in the design and outcomes, leading to a poor understanding of the relationship between the two. Although IDR are increasingly being used, to our knowledge, there is no published evidence regarding the optimal composition of IDR teams or how specific outcomes may be impacted by team composition or focus. This is a particular problem in general medicine units caring for patients with complex medical and social issues whose care involves several professionals. In addition, the results from other IDR settings may not be transferable to general medicine units.

Therefore, we conducted a systematic review of experimental, quasiexperimental, and observational studies to (1) document types of IDR on general medicine units, (2) categorize IDR interventions by similarities in team composition and focus, and (3) determine the differential impact of each category of intervention on outcomes including measures of efficiency, quality, safety, and satisfaction.

\section{METHODS}

This systematic review was performed according to the Preferred Reporting Items for Systematic Reviews and Meta-Analyses (PRISMA) guidelines. ${ }^{8}$ 


\section{Data Sources and Searches}

We conducted systematic literature searches of databases including Ovid MEDLINE, Ovid MEDLINE InProcess \& Other Non-Indexed Citations, Journals@Ovid, Cumulative Index to Nursing and Allied Health Literature (EBSCOhost), and PubMed (NCBI/National Library of Medicine) to identify English-language articles published from 1990 to 2014. In Ovid MEDLINE, the librarians (E.M.J., E.B.) identified a combination of relevant Medical Subject Headings and keywords to capture the concepts of interdisciplinary rounds and general medicine hospital units. To identify additional relevant studies, we examined reference lists from included studies and review articles. A detailed search strategy for Ovid MEDLINE is included in the Supporting Information, Appendix A, in the online version of this article.

\section{Study Selection}

One author (V.S.B.) screened titles for abstract selection. Two reviewers (D.J.E. and V.S.B.) independently reviewed all abstracts for full-text eligibility. A third reviewer adjudicated all inclusion disagreements (E.J.R.).

We included IDR studies where the attending physician or resident physician and at least one other healthcare team member (from a different discipline) managing a common group of patients was present. We used this as a screening criterion rather than a definition of IDR to include studies that would be relevant to the current climate in inpatient medicine. Although there is no accepted definition of IDR, IDR are generally designed as a process that involves several team members. However, we included studies that utilized fewer team members for completeness and to investigate possible linkages between design and outcomes. We included experimental, quasiexperimental, and observational studies on general medicine units in the English-language literature. We were neutral to cardiac monitoring status and age of general medicine patients. We excluded studies lacking a definite IDR intervention or a study design. We excluded health care settings other than inpatient medicine, and intensive care units (ICUs) were excluded. A flow diagram outlining the study selection process appears as Supporting Information, Appendix B, in the online version of this article.

\section{Data Extraction and Study Quality Assessment}

We drafted an abstraction tool based on published reports of IDR. ${ }^{9,10}$ Three reviewers (V.S.B., D.J.E., and E.J.R.) independently tested the tool's applicability to several included articles. We developed the tool in an iterative process to come up with a final version by reviewer consensus. Two reviewers (V.S.B., S.S.S.) abstracted all articles. Disagreements were resolved through consensus.
We categorized abstraction elements into three categories: (1) study setting and characteristics, (2) IDR design, and (3) IDR outcomes. Study setting and characteristics included setting and location, type of unit, study design, and number of study participants (intervention vs control groups) when available. The IDR design category included timing, location, duration, and frequency of rounds, time per patient, presence of geographic colocation of physician's patients (geographic cohorting), use of team training for IDR teams, format of IDR (scripted vs free-flowing discussion), use of patient communication tools, and use of safety checklists. Team composition was also included in the IDR design category. This included attending physician, bedside nurse, nurse leader or charge nurse, case manager, pharmacist, social worker, resident, and/or medical student. Some studies referenced a nurse or nurse leader who facilitated rounds, which we collected as a rounds manager, based on IHI recommendations. We were also interested in patient and family presence in rounds and documented such when available. The IDR outcomes category included hospital length of stay (LOS), cost per case, use of cardiac monitors, readmission rates, rates of venous thromboembolism:prophylaxis and occurrence, falls, skin breakdown, hospitalacquired infections, and patient and staff satisfaction.

We modified the 27-question Downs and Black quality scoring tool ${ }^{11}$ to include 15 questions aligned with study characteristics relevant to IDR (see Supporting Information, Appendix $\mathrm{C}$, in the online version of this article). Scoring was yes/no (1/0) for each quality indicator, allowing scores from 0 to 15 . We categorized studies with scores 0 to 5 as low, 6 to 10 as medium, and 11 to 15 as high-quality studies. Two reviewers (V.S.B. and S.S.S.) independently performed quality scoring of all articles, and disagreements were resolved through consensus.

\section{Data Synthesis and Analysis}

Due to significant variability in IDR characteristics, design and outcomes, a meta-analysis was not feasible. As a result, we did a narrative review of IDR design and outcomes. To understand the potential causal pathways that relate IDR design to outcomes, we grouped studies with similar design and explored similarities in outcomes in those groups. We report the number of studies both as a number and percentage within each subgroup rounded to the nearest lower whole number.

\section{RESULTS}

The searches identified 12,692 titles. We eliminated duplicates and applied inclusion and exclusion criteria to titles and abstracts, leading to review of 259 fulltext articles. Hand searching yielded two additional titles. Of these, 239 articles were excluded, leaving 22 full-text articles for abstraction. Study setting and characteristics appear as Table 1. 
IDR in Hospitalized Medicine Patients | Bhamidipati et al

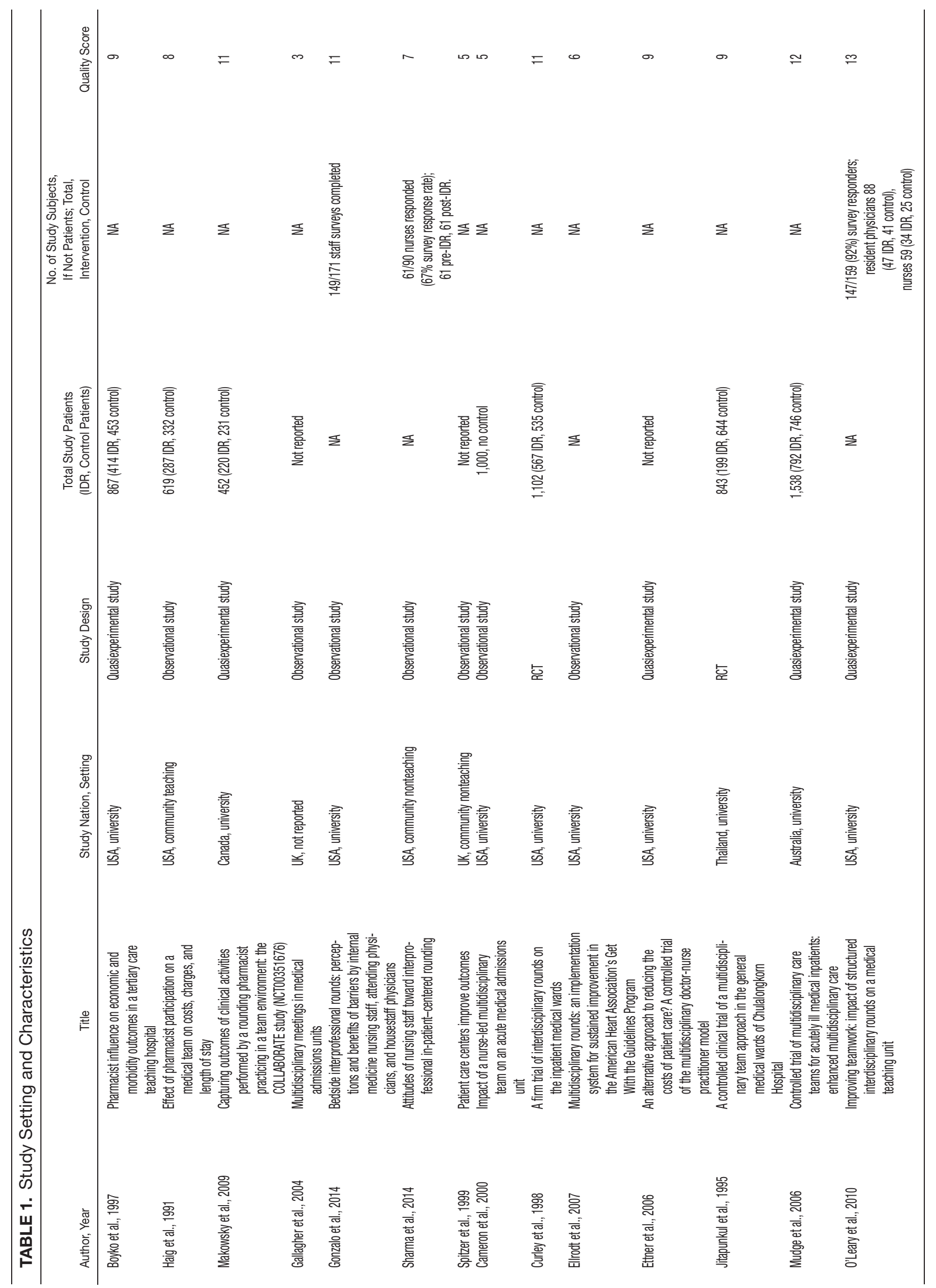




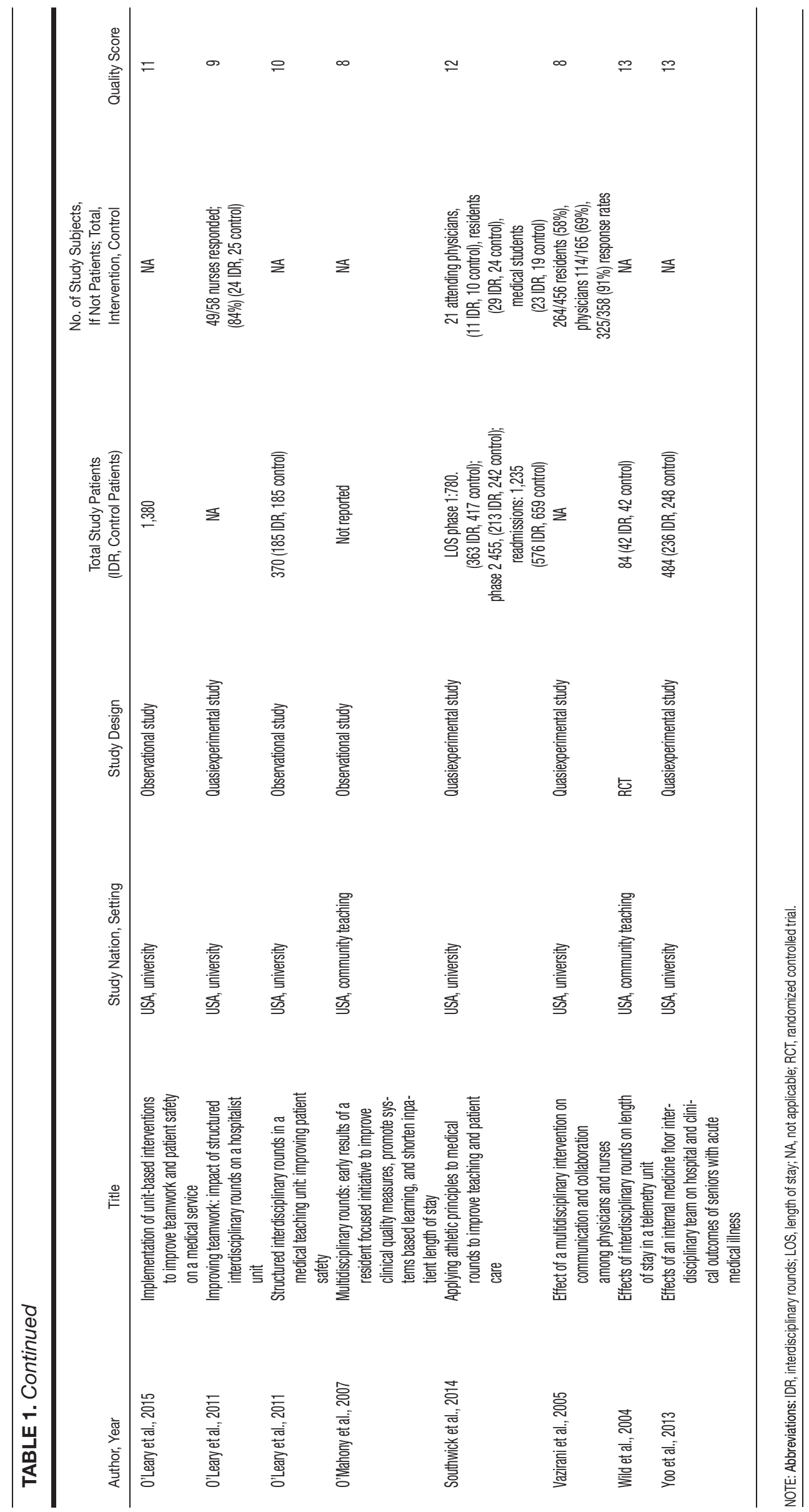




\section{IDR Design}

There were three areas of focus identified: pharmacist studies, bedside rounding studies, and interdisciplinary team studies. Table 2 summarizes IDR team composition and design features.

\section{Pharmacist Studies (13\% of All Studies)}

The three studies in this group were characterized by a physician-resident team rounding with a pharmacist. ${ }^{12-14}$ Pharmacist recommendations were incorporated into patient plans of care.

\section{Bedside Rounding Studies (18\% of All Studies)}

The four studies in this group were characterized by bedside rounding as a team with patients. ${ }^{15-18}$ All four studies included patient and family as partners in determining plans of care. Two studies ${ }^{15,16}(50 \%)$ described physician and nurse bedside rounding, whereas the other two ${ }^{17,18}(50 \%)$ included a larger complement of team members, notably a discharge planner. Timing, duration, use of IDR scripts, and team training were not reported.

\section{Interdisciplinary Team Studies (68\% of All Studies)}

The 15 studies in this group were characterized by two or more team members rounding with a physician. ${ }^{9,10,19-31}$ Thirteen studies $(86 \%)$ reported rounding once a day in the morning, often restricted to weekdays only. ${ }^{9,14,25,27}$ Only four $(26 \%)$ studies $^{19,20,23,31}$ reported rounding time per patient. Eight $(53 \%)$ studies $9,21,24,27-31$ reported geographic physician-patient colocation. Ten $(66 \%)$ studies $9,21-24,27-31$ reported training teams. Nine $(60 \%)$ studies ${ }^{10,20,21,23,24,28-31}$ reported a scripted discussion during rounds, with adherence to script measured in only two $(13 \%)$ studies. ${ }^{21,28}$ Four $(26 \%)$ studies ${ }^{28-31}$ reported using a safety checklist. Nurses, pharmacists, social workers, and case managers were the most common participants in IDR. Roles and responsibilities of individual team members were inconsistently described. Particularly, the role of case manager and social worker were not clearly defined, although it appeared that both roles contributed to discharge planning. Ten $(66 \%)$ studies $^{9,20,23,25,27-31}$ reported an individual (usually a nurse or nurse leader) present as a manager and coach for rounds.

\section{IDR Outcomes and Relationship Between Design and Outcomes}

We report IDR outcomes within each IDR design group. Table 2 summarizes IDR design and outcomes.

\section{Pharmacist Studies}

All three studies in this group were of medium quality. ${ }^{12-14} \mathrm{Two}^{12,13}(66 \%)$ reported a reduction in LOS. Two studies ${ }^{12,13}(66 \%)$ reported a reduction in cost but used different definitions for cost. Boyko et al. ${ }^{13}$ (defined as hospital costs) and Haig et $\mathrm{al}^{12}$ (defined as hospital charges) studies reported a decrease in both pharmacy and total costs. Only one study ${ }^{14}$ (33\%) reported a decrease in readmission rates and a concomitant rise in LOS. Review of these studies suggests a relationship between pharmacist-physician rounding and decrease in cost and LOS.

\section{Bedside Rounding Studies}

Only one $\mathrm{e}^{16}(25 \%)$ of the four studies is a high-quality study. ${ }^{15-18}$ Three studies $^{15-17}(75 \%)$ focused on nurse-physician bedside rounding. Only one study ${ }^{17}$ reported patient satisfaction, which was measured using a local survey. Two studies ${ }^{15,16}(50 \%)$ reported increased satisfaction for rounding team members by both physicians and nurses. One ${ }^{18}(25 \%)$ utilized a complement of team members, including a discharge planner at the bedside, and reported a decrease (not statistically significant) in LOS. These studies suggest (1) a relationship between bedside rounding and patient and team satisfaction and (2) large rounding team (possibly with a discharge planner) and efficiency.

\section{Interdisciplinary Team Studies}

Of the 15 interdisciplinary team studies, ${ }^{9,10,19-31}$ there were seven high-quality studies ${ }^{10,19,21,22,24,28,30}$ (46\%). LOS, cost, harm reduction, and patient and staff satisfaction are the commonly reported outcomes.

\section{LOS}

Five $(33 \%)$ studies $^{20-22,24,26}$ reported a statistically significant decrease in LOS. Several of these studies utilized either a case manager $20,21,24$ or a social worker 22,26 in a discharge planning role. In these studies, physicians rounded with at least two but mostly three team members. Three $1,22,24 \quad(20 \%)$ of the LOS studies were of high quality, were done on teaching units, and included a large complement of team members including a discharge planner. All three studies also trained teams to participate in IDR. One study ${ }^{21}$ was a two-phase study that demonstrated additional decrease in LOS after utilizing a case manager and training teams in communication. Two ${ }^{10,31}(13 \%$; one medium and one high quality) other studies in this group that were designed similar to the above three studies used a large complement of team members, including a discharge planner and trained teams, but did not report LOS reduction. Overall, the results from the high-quality studies point to larger teams, discharge planners, and team training as notable features possibly linked to LOS reduction.

\section{Cost}

Two $(13 \%)$ of the 15 studies $^{24,27}$ reported a decrease in cost per case, defined as hospital costs in the Ettner et al. study ${ }^{27}$ and hospital charges in the Curley et al. ${ }^{24}$ study. The Curley et al. study included a pharmacist similar to the studies ${ }^{12,13}$ in the pharmacist group. This led to the possibility that pharmacist 


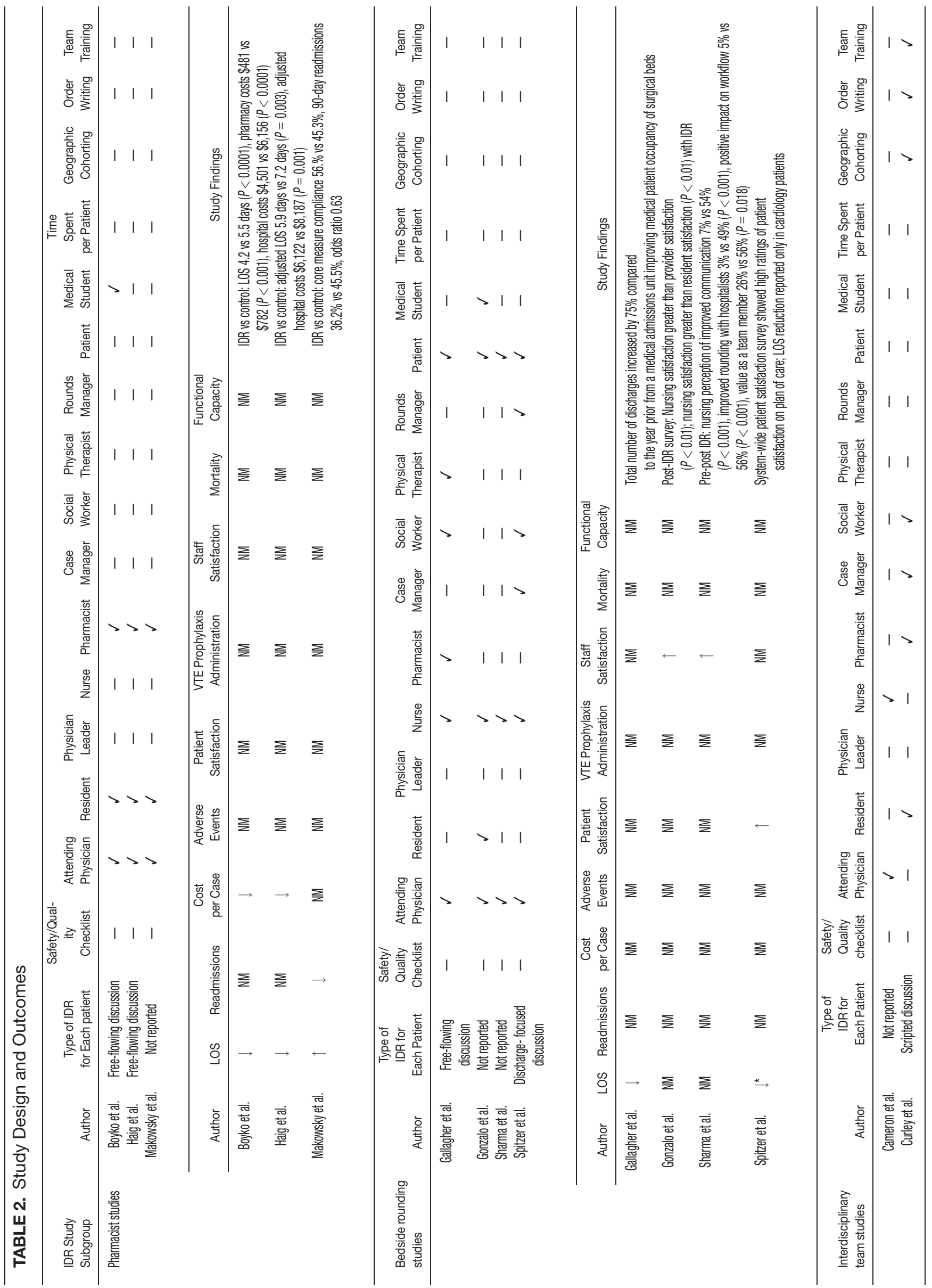




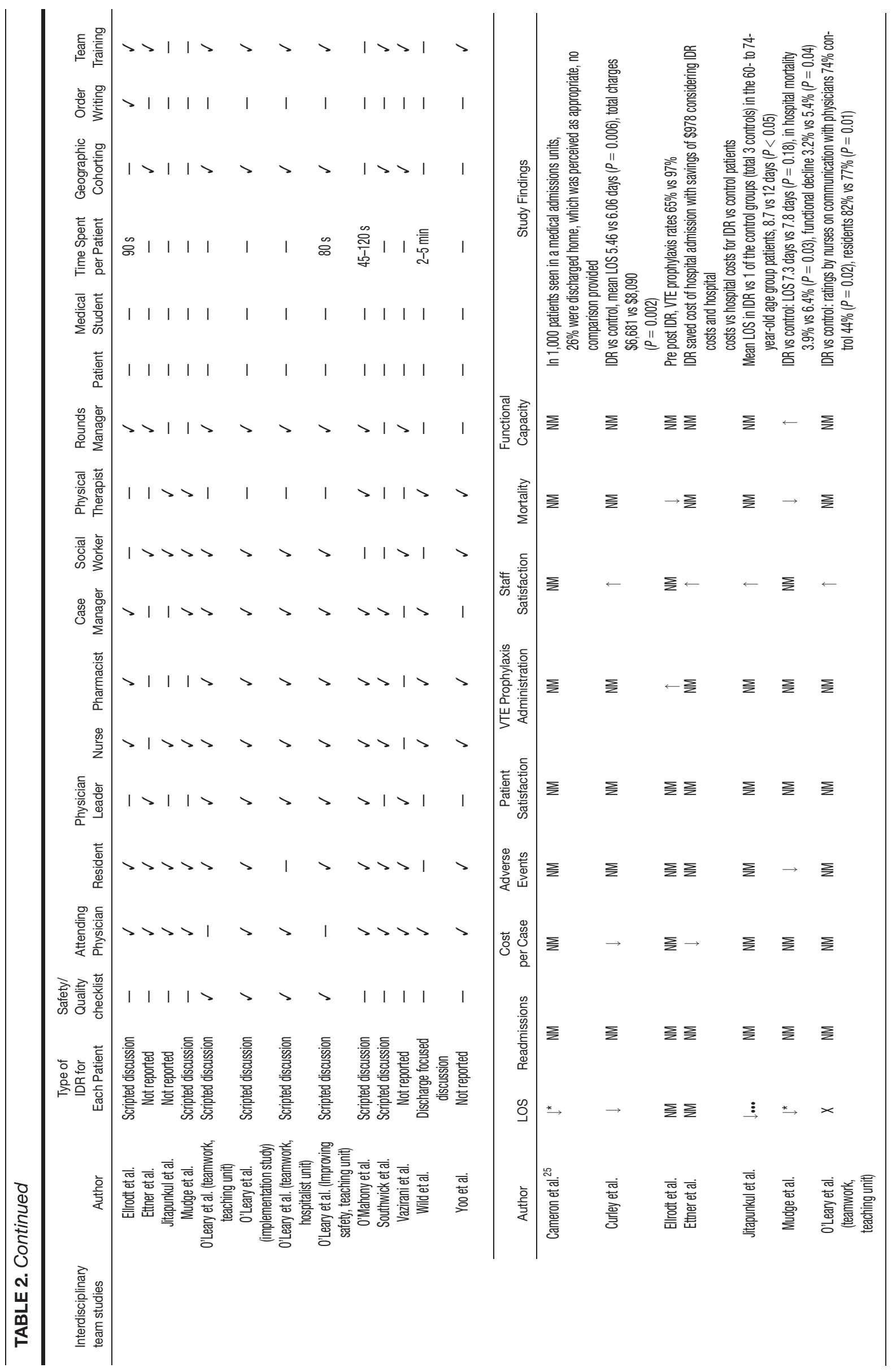




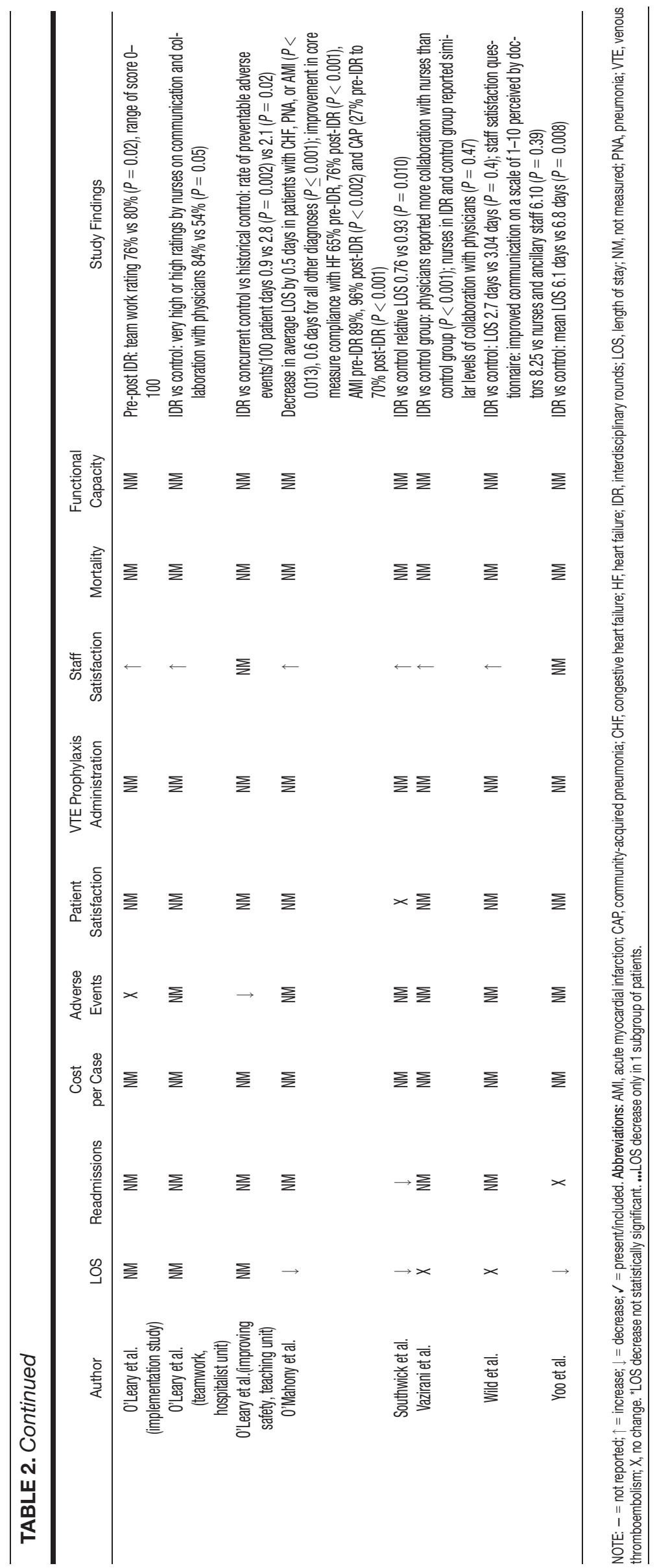


presence in IDR could influence cost reductions. This hypothesis could have been more definitive if the several other studies ${ }^{20-22}$ that utilized a pharmacist also measured cost.

\section{Harm Reduction}

Only three $(20 \%)$ studies ${ }^{10,23,31}$ reported reduction in patient harm as a result of IDR. Utilization of safety and quality checklists ${ }^{28,31}$ did not reliably demonstrate a decrease in adverse events. Two studies ${ }^{10,23}$ $(13 \%)$ reported a decrease in mortality. Both studies had a large complement of team members, but we could not isolate any specific features in their model that would link their IDR design to outcomes.

\section{Patient Satisfaction}

Only one $(6 \%)$ study $^{10}$ in this group reported improving patient satisfaction with IDR. This study did not include patients in IDR. With this being the only study in this group that reported patient satisfaction, we could not identify an IDR feature that could have led to improved patient satisfaction.

\section{Staff Satisfaction}

Although staff satisfaction has not been clearly linked to clinical outcomes, conceptual models ${ }^{32}$ have been proposed linking staff satisfaction to patient reported outcomes. Several studies $(71 \%)$ measured and reported improvement ${ }^{9,19-21,24,26-28,30,31}$ in staff satisfaction (all participants). Some studies reported more nursing satisfaction than physician, ${ }^{16}$ and some reported more physician satisfaction than nurse. ${ }^{19}$ Rounds manager, team training, and geographic cohorting were commonly reported in many of these studies. 9,27,29-31 However, we did not see a specific IDR model that could be linked to staff satisfaction.

\section{DISCUSSION}

In a systematic review of the literature on IDR in general medicine units, we found significant variability in IDR design, outcomes, and reporting. We found 3 different models of IDR: pharmacist focused, bedside rounding, and interdisciplinary team studies. There are data to suggest a relationship between IDR and improvements in LOS and staff satisfaction but little data on patient safety or satisfaction. Our review did not reveal clear causal pathways between IDR design and outcomes but allowed for generation of some hypotheses that require further testing:

- Physician-pharmacist rounding may be related to decrease in LOS and cost.

- Presence of discharge planner, team training, and large complement of team members may be related to LOS reduction.

- Physician-nurse or team rounding in general may be related to staff satisfaction.

The reviewed studies underscore the absence of a standardized definition of IDR, with no common pro- cess or outcome measures across studies. Few studies provided complete information on design, and even fewer reported similar outcomes, making it difficult to identify links between IDR characteristics and outcomes. As a result, we provide recommendations for an IDR definition and suggested future taxonomy studies (Table 3).

Several studies $(59 \%)$ were interested in LOS. From the high-quality studies $^{21,22,24}$ that reported LOS reductions, it is notable that large teams, discharge planner presence, and team training are common features. This may be worth further investigation when focused on using IDR to decrease LOS, particularly in community settings, as these studies were done in academic institutions. Real-time input from several team members, presence of a discharge planner, and highly effective teams could be a potential causal pathway to increased unit efficiency but should be rigorously tested.

All four studies $12,13,24,27$ that reported decreased hospital costs utilized a pharmacist, with three ${ }^{12,13,24}$ of the four also reporting decreased LOS. Decreasing medication utilization and costs through pharmacist participation in IDR, as well as a decrease in LOS, could explain the hospital cost decreases found in these studies. Overall, it appears that pharmacist interventions tend to focus on cost and utilization.

It appears that geographic cohorting, team training, and utilizing a rounds manager are common features in studies that report staff satisfaction. ${ }^{9,27-31}$ Although we cannot draw any conclusions from this finding, the association can be used to generate a hypothesis. Although staff satisfaction could conceivably be improved through the improved communication inherent in IDR, it is also possible that team efficiency and satisfaction is further enhanced by geographic cohorting, team training, and utilizing a rounds manager.

The role of safety checklists remains unclear, as the gains demonstrated in the O'Leary et al. study ${ }^{31}$ were not replicable, as the IDR intervention expanded ${ }^{28}$ to several other units in their institution. The role of IDR in preventing adverse events is also unclear.

Although we were interested in patient and family participation and patient-reported outcomes, in the bedside rounding studies, ${ }^{15-18}$ only one study ${ }^{17}$ measured patient satisfaction. Overall, this review revealed limited data ${ }^{10,17}$ on patient satisfaction due to IDR. As a result, the relationship between patient and family participation in IDR and outcomes remains unclear and needs further study.

This review has limitations. Due to the small sample sizes and inconsistent reporting of data among studies, we had insufficient power for a $\chi^{2}$ analysis to generate meaningful meta-analytic results. Our search strategy, although inclusive, could have missed articles, so we compensated by manual searches. Selection of outcome-driven studies could have eliminated quality improvement reports. Lack of 
TABLE 3. Proposed IDR Definition and Suggested Taxonomy for Future Studies

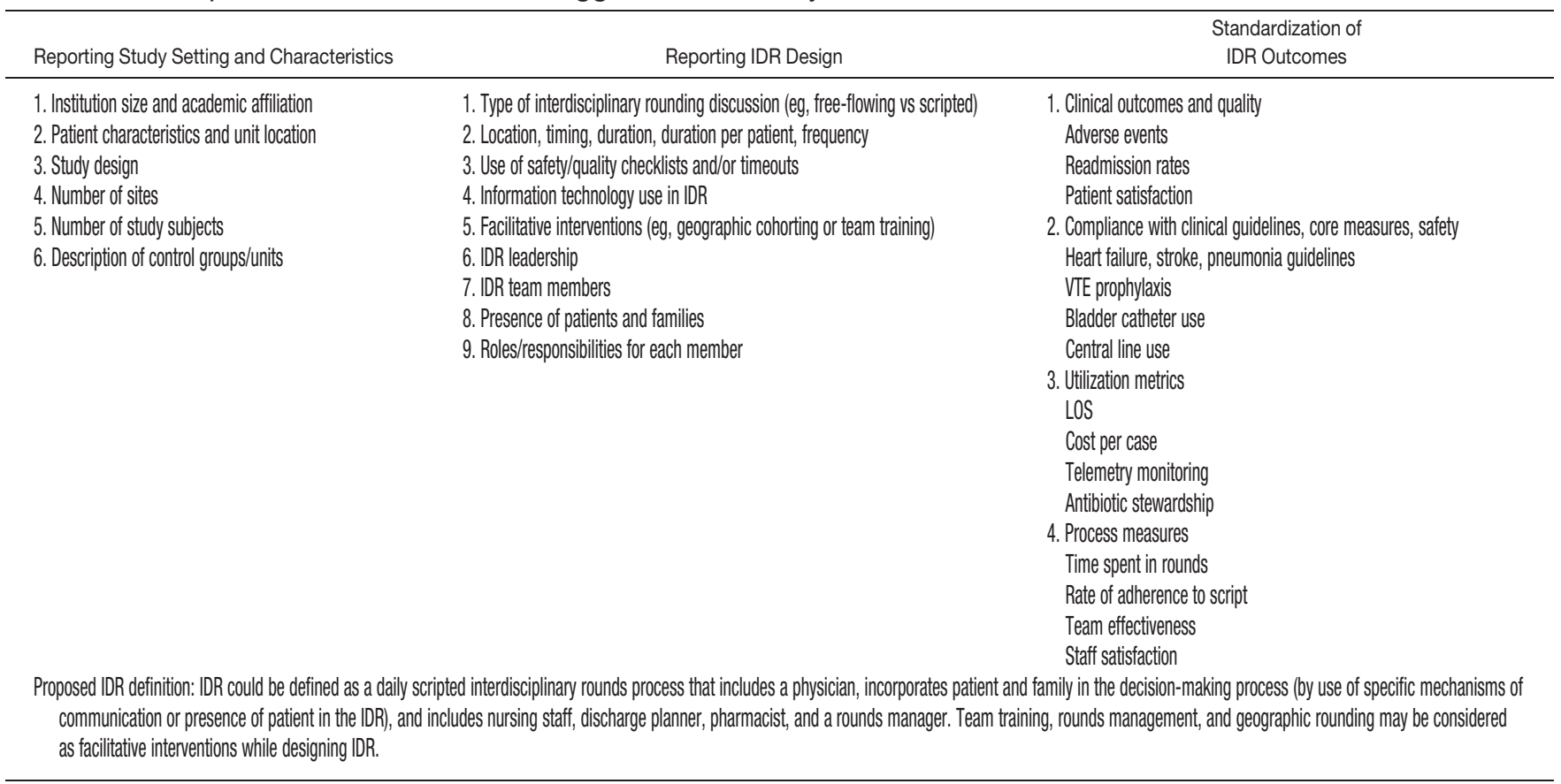

NOTE: Abbreviations: IDR, interdisciplinary rounds; LOS, length of stay; VTE, venous thromboembolism.

publications of negative studies is also a potential problem that could have biased the review toward the positive impact of IDR interventions. Lastly, although the Downs and Black scoring tool is validated, our modified version has not been validated.

\section{CONCLUSIONS}

Our review revealed that IDR may be an important tool for improving efficiency and staff satisfaction, with the potential to improve safety. However, more deliberately designed and completely reported studies are needed to fully understand optimal IDR design. Given the difficulties of implementing robust, randomized, and controlled studies in this setting, standardizing the design and reporting elements of IDR is necessary to inform decision making surrounding the development, implementation, and proposed expansion of these programs. In Table 3 we propose an IDR definition and suggested taxonomy for future studies.

\section{Acknowledgements}

The authors acknowledge the support and insightful feedback of Dr. LeRoi Hicks in the preparation of this article.

Disclosure: Nothing to report.

\section{References}

1. Halm MA, Gagner S, Goering M, Sabo J, Smith M, Zaccagnini M. Interdisciplinary rounds: impact on patients, families, and staff. Clin Nurse Spec. 2003;17(3):133-142.

2. Burger CD. A method to improve quality and safety of critically ill patients. Northeast Fla Med. 2007;58(3):16-19.

3. Pannick S, Beveridge I, Wachter RM, Sevdalis N. Improving the quality and safety of care on the medical ward: a review and synthesis of the evidence base. Eur J Intern Med. 2014;25(10):874-887.
4. Kucukarslan SN, Peters M, Mlynarek M, Nafziger DA. Pharmacists on rounding teams reduce preventable adverse drug events in hospital general medicine units. Arch Intern Med. 2003;163(17):20142018.

5. Kim MM, Barnato AE, Angus DC, Fleisher LA, Fleisher LF, Kahn JM. The effect of multidisciplinary care teams on intensive care unit mortality. Arch Intern Med. 2010;170(4):369-376.

6. Bharwani AM, Harris GC, Southwick FS. Perspective: a business school view of medical interprofessional rounds: transforming rounding groups into rounding teams. Acad Med. 2012;87(12):1768-1771.

7. Institute for Healthcare Improvement. How-to guide: multidisciplinary rounds. Available at: http://www.ihi.org/resources/Pages/Tools/ HowtoGuideMultidisciplinaryRounds.aspx. Published 2010. Accessed January 1, 2015.

8. Preferred Reporting Items for Systematic Reviews and Meta-Analyses. PRISMA statement. Available at: http://prisma-statement.org/. Accessed November 23, 2015.

9. Vazirani S, Hays RD, Shapiro MF, Cowan M. Effect of a multidisciplinary intervention on communication and collaboration among physicians and nurses. Am J Crit Care. 2005;14(1):71-77.

10. Mudge A, Laracy S, Richter K, Denaro C. Controlled trial of multidisciplinary care teams for acutely ill medical inpatients: enhanced multidisciplinary care. Intern Med J. 2006;36(9):558-563.

11. Downs SH, Black N. The feasibility of creating a checklist for the assessment of the methodological quality both of randomised and non-randomised studies of health care interventions. J Epidemiol Community Health. 1998;52:377-384.

12. Haig GM, Kiser LA. Effect of pharmacist participation on a medical team on costs, charges, and length of stay. Am J Hosp Pharm. 1991; 48(7):1457-1462.

13. Boyko WL, Yurkowski PJ, Ivey MF, Armitstead JA, Roberts BL. Pharmacist influence on economic and morbidity outcomes in a tertiary care teaching hospital. Am J Health Syst Pharm. 1997;54(14):1591-1595.

14. Makowsky M, Koshman S. Capturing outcomes of clinical activities performed by a rounding pharmacist practicing in a team environment: the COLLABORATE study. Med Care. 2009;47(6):642-650.

15. Sharma U, Klocke D. Attitudes of nursing staff toward interprofessional in-patient-centered rounding. J Interprof Care. 2014;1820(5): $475-477$.

16. Gonzalo JD, Kuperman E, Lehman E, Haidet P. Bedside interprofessional rounds: perceptions of benefits and barriers by internal medicine nursing staff, attending physicians, and housestaff physicians. J Hosp Med. 2014;9(10):646-651.

17. Spitzer WJ, Savage L, Weihl B, Baker B, Miller W, McClain M. Patient care centers improve outcomes. Continuum. 1999;19(1):14-19.

18. Gallagher A, Lynch D. Multidisciplinary meetings in medical admissions units. Nurs Times. 2004;100(44):34-36. 
19. Wild D, Nawaz H, Chan W, Katz DL. Effects of interdisciplinary rounds on length of stay in a telemetry unit. J Public Health Manag Pract. 2004;10(1):63-69.

20. O’Mahony S, Mazur E, Charney P, Wang Y, Fine J. Use of multidisciplinary rounds to simultaneously improve quality outcomes, enhance resident education, and shorten length of stay. J Gen Intern Med. 2007;22(8):1073-1079.

21. Southwick F, Lewis M, Treloar D, et al. Applying athletic principles to medical rounds to improve teaching and patient care. Acad Med. 2014;89(7):1018-1023.

22. Yoo JW, Kim S, Seol H, et al. Effects of an internal medicine floor interdisciplinary team on hospital and clinical outcomes of seniors with acute medical illness. Geriatr Gerontol Int. 2013;13:942-948.

23. Ellrodt G, Glasener R, Cadorette B, et al. Multidisciplinary rounds (MDR): an implementation system for sustained improvement in the American Heart Association's Get With The Guidelines program. Crit Pathw Cardiol. 2007;6(3):106-116.

24. Curley C, McEachern JE, Speroff T. A firm trial of interdisciplinary rounds on the inpatient medical wards: an intervention designed using continuous quality improvement. Med Care. 1998;36(8 suppl):AS4AS12.

25. Cameron S, McKenzie F, Warnock L, Farquhar D. Impact of a nurse led multidisciplinary team on an acute medical admissions unit. Health Bull (Edinb). 2000;58(6):512-514.
26. Jitapunkul S, Nuchprayoon C, Aksaranugraha S, et al. A controlled clinical trial of multidisciplinary team approach in the general medical wards of Chulalongkorn Hospital. J Med Assoc Thai. 1995;78(11):618-623.

27. Ettner SL, Kotlerman J, Afifi A, et al. An alternative approach to reducing the costs of patient care? A controlled trial of the multidisciplinary doctor-nurse practitioner (MDNP) model. Med Decis Making. 2006;26(1):9-17.

28. O'Leary KJ, Creden AJ, Slade ME, et al. Implementation of unitbased interventions to improve teamwork and patient safety on a medical service. Am J Med Oual. 2015;30(5):409-416.

29. O'Leary KJ, Haviley C, Slade ME, Shah HM, Lee J, Williams MV. Improving teamwork: impact of structured interdisciplinary rounds on a hospitalist unit. J Hosp Med. 2011;6(2):88-93.

30. O'Leary KJ, Wayne DB, Haviley C, Slade ME, Lee J, Williams M V. Improving teamwork: impact of structured interdisciplinary rounds on a medical teaching unit. J Gen Intern Med. 2010;25(8): 826-832.

31. O'Leary KJ, Buck R, Fligiel HM, et al. Structured interdisciplinary rounds in a medical teaching unit. Arch Intern Med. 2011;171(7): 678-684.

32. Scotti DJ, Harmon J, Behson SJ. Links among high-performance work environment, service quality, and customer satisfaction: an extension to the healthcare sector. I Healthc Manag. 52(2):109-124; discussion $124-125$. 